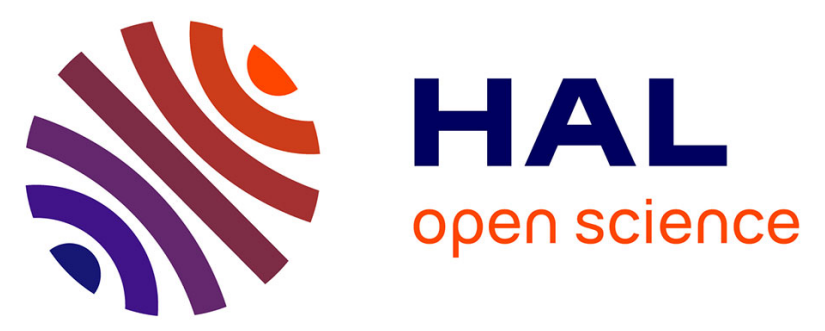

\title{
Utilisation du protocole de surveillance en entreprise : expérience du programme de surveillance épidémiologique des TMS dans les Pays de la Loire
}

Yves Roquelaure, Catherine Ha, Annie Touranchet, Alexis Descatha, Patrick

Bidron, B. Lendevic, François Leroux, Annick Mazoyer, Françoise Méritet, Marcel Goldberg, et al.

\section{To cite this version:}

Yves Roquelaure, Catherine Ha, Annie Touranchet, Alexis Descatha, Patrick Bidron, et al.. Utilisation du protocole de surveillance en entreprise : expérience du programme de surveillance épidémiologique des TMS dans les Pays de la Loire. Archives des Maladies Professionnelles et de L'Environnement, 2010, 71 (3), pp.420 - 423. 10.1016/j.admp.2010.03.075 . hal-03389903

\section{HAL Id: hal-03389903 \\ https://univ-angers.hal.science/hal-03389903}

Submitted on 21 Oct 2021

HAL is a multi-disciplinary open access archive for the deposit and dissemination of scientific research documents, whether they are published or not. The documents may come from teaching and research institutions in France or abroad, or from public or private research centers.
L'archive ouverte pluridisciplinaire HAL, est destinée au dépôt et à la diffusion de documents scientifiques de niveau recherche, publiés ou non, émanant des établissements d'enseignement et de recherche français ou étrangers, des laboratoires publics ou privés. 


\section{Utilisation du protocole de surveillance en entreprise : expérience du programme de surveillance épidémiologique des TMS dans les Pays de la Loire}

Use of a hazard monitoring protocol at the workplace: The French musculoskeletal disorders surveillance program and the Pays de Loire Network

Y. Roquelaure ${ }^{a *}$, C. Ha ${ }^{b}$, A. Touranchet ${ }^{c}$, A. Descatha ${ }^{d}$, P. Bidron ${ }^{e}$, B. Lendevic ${ }^{e}$,

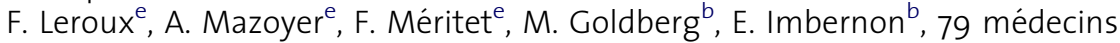
du travail de la région des Pays de la Loire

a IFR 132, UPRES EA 4336, unité associée à l'institut de veille sanitaire, laboratoire d'ergonomie et d'épidémiologie en santé au travail (LEEST), CHU d'Angers, université d'Angers, 49933

Angers cedex, France

${ }^{\mathrm{b}}$ Département santé travail, institut de veille sanitaire, Saint-Maurice, France

${ }^{\mathrm{C}}$ DRTEFP Pays de la Loire, Nantes, France

dInserm U687, Villejuif, France

$\checkmark$ eservices de santé au travail des Pays de la Loire, Nantes, France

es troubles musculo-squelettiques des membres supérieurs (TMS-MS) resteront, avec les rachialgies, l'un des enjeux majeurs de la santé au travail des années à venir en raison de leur augmentation probable par conjugaison d'une intensification des conditions de travail et du vieillissement de la population active. Le programme pilote de surveillance épidémiologique des TMS mis en place en 2002 dans la région des Pays de la Loire par l'institut de veille sanitaire [8] a permis d'estimer les prévalences des TMS et de leurs facteurs de risque en population salariée $[2,17,18]$.

Ce programme utilise pour la première fois en Europe les définitions des TMS-MS et la démarche diagnostique standardisée proposées par le consensus européen Saltsa pour la surveillance des TMS-MS $[7,13,15,20]$.

L'objectif de cet article est de décrire le protocole du programme TMS et de discuter la faisabilité de l'utilisation en France d'un protocole européen de surveillance des TMS-MS.

\footnotetext{
Auteur correspondant.

e-mail : YvRoquelaure@chu-angers.fr
}

\section{Méthodes}

Cette surveillance épidémiologique des TMS en entreprise a été mise en œuvre entre 2002 et 2004 dans les cinq départements de la région des Pays de la Loire, grâce à la participation de 83 médecins du travail volontaires, représentant $18 \%$ des médecins du travail de la région. Au total, 3710 salariés âgés de 20 à 59 ans ( 2162 hommes et 1548 femmes, âge moyen $=38,4$ $\pm 10,3$ ans) ont été inclus par tirage au sort. L'échantillon est globalement représentatif des salariés des entreprises privées et publiques des Pays de la Loire [17].

Les symptômes musculo-squelettiques des membres et du rachis ont été recueillis à l'aide d'un autoquestionnaire dérivé du questionnaire scandinave dit "nordique " [11]. Les six principaux TMS-MS (syndrome de la coiffe des rotateurs, épicondylite latérale, tendinites des fléchisseurs ou des extenseurs des doigts, ténosynovite de De Quervain, syndrome du canal carpien, syndrome du tunnel cubital) ont été diagnostiqués par les médecins du travail à l'aide des manœuvres cliniques standardisées et des arbres diagnostiques proposés par le consensus Saltsa $[20,13]$. Les médecins ont été préalablement formés à la théorie et à la pratique de cette démarche diagnostique au cours de sessions organisées dans chaque

$1775-8785 \mathrm{X} / \$$ - see front matter (c) 2010 Elsevier Masson SAS. Tous droits réservés. 
service de médecine du travail. Les formateurs étaient les coordonnateurs du programme pilote assistés de deux internes en médecine du travail. Le protocole et les manœuvres cliniques illustrées par des tableaux et photos ainsi que les diagnostics différentiels à évoquer ont été publiés [7]. Les données d'exposition professionnelle ont été recueillies à l'aide d'un autoquestionnaire portant sur les facteurs de risque de TMS biomécaniques (répétitivité, force, postures, vibrations), psychosociaux (demande psychologique, soutien social au travail, latitude décisionnelle du modèle de Karasek et Theorell) et organisationnels. Ce questionnaire a été développé par nous-mêmes, d'une part, à partir des définitions des facteurs de risque proposées par le consensus Saltsa pour le membre supérieur et Halpern et al. [10] pour le rachis et, d'autre part, des grandes enquêtes françaises sur les TMS et les conditions de travail (Estev, Sumer, Dares, Inserm-Anact) [15]. La prévalence des symptômes et des pathologies a été estimée par sujet. Des scores d'exposition au risque de TMS ont été calculés selon les recommandations du consensus européen par sommation du nombre de facteurs de risque auxquels est exposé le salarié. En première analyse, l'exposition professionnelle a été classée faible ou en "zone verte ", modérée ou en "zone jaune ", élevée ou en "zone rouge " selon que le salarié était exposé respectivement à aucun, un ou au moins deux facteurs de risque [20].

\section{Résultats}

\section{Prévalence des six principaux TMS des membres supérieurs}

Au moins un TMS-MS a été diagnostiqué par le médecin du travail chez $15 \%$ des femmes et $11 \%$ des hommes. Le syndrome de la coiffe des rotateurs est le plus fréquent ( $9 \%$ des femmes et $7 \%$ des hommes), devant le syndrome du canal carpien ( $4 \%$ des femmes et $2 \%$ des hommes) et l'épicondylite latérale (3\% des femmes et $2 \%$ des hommes). Chez les salariés âgés de 50 à 59 ans, près de $26 \%$ des femmes et $22 \%$ des hommes souffrent d'un TMS-MS et $6 \%$ des femmes et $4 \%$ des hommes d'au moins deux. La prévalence des TMS-MS est inégalement répartie en fonction des secteurs d'activité et des catégories socioprofessionnelles. Les secteurs d'activité les plus touchés sont, d'une part, les secteurs industriels, (notamment l'automobile, les industries des biens intermédiaires et des biens de consommation, l'agro-alimentaire) et, d'autre part, l'agriculture, les transports et l'administration. Les catégories ouvrières, notamment les ouvriers non qualifiés de l'industrie, les ouvriers agricoles et les manutentionnaires, sont les plus touchées par les TMS-MS, quel que soit l'âge et le sexe, devant les employés.

\section{Prévalence de l'exposition au risque de TMS}

La majorité des salariés des deux sexes est fortement exposée aux facteurs de risque professionnels de TMS-MS, qu'ils soient biomécaniques, psychosociaux et organisationnels. Les salariés les plus jeunes (20-29 ans) sont les plus exposés : $72 \%$ d'entre eux sont exposés à deux facteurs de risque ou plus contre $65 \%$ des 30 à 39 ans, $63 \%$ des 40 à 49 ans et $62 \%$ des 50 à 59 ans. La surexposition des plus jeunes s'explique principalement par la surexposition des travailleurs intérimaires (13,9\% des moins de 30 ans sont intérimaires contre $2,7 \%$ des plus de 30 ans). L'exposition professionnelle au risque de TMS-MS reste élevée pour les salariés âgés de 50 ans et plus tandis que leurs capacités fonctionnelles diminuent. Cela est vrai pour les principaux facteurs de risque tels que la répétitivité des gestes, la force, les postures inconfortables. Le manque de latitude décisionnelle pour faire face aux contraintes de la tâche concerne une majorité de salariés de 50 ans et plus : plus d'un homme sur deux et sept femmes sur dix. Le niveau de l'exposition aux facteurs de risque de TMS-MS est particulièrement élevé pour les catégories ouvrières et, à un moindre degré, pour les employés. Il est moins élevé pour les professions intermédiaires et pour les cadres. Après 50 ans, les trois-quarts des ouvriers restent exposés à un risque élevé de TMS-MS.

\section{Discussion}

\section{La surveillance épidémiologique des TMS}

La surveillance épidémiologique des TMS mise en œuvre dans les Pays de la Loire était basée sur un réseau de 83 médecins sentinelles dont les caractéristiques professionnelles étaient comparables à celles des médecins non participants. L'inclusion aléatoire des salariés a permis d'assurer une représentativité satisfaisante de l'échantillon par rapport à la population salariée régionale [17], fournissant une meilleure estimation de la prévalence des TMS-MS parmi les salariés que les études françaises préalablement conduites dans des secteurs à haut risque de TMS [12].

Cette surveillance a fourni des données précises et inédites sur la fréquence des TMS dans un échantillon représentatif de salariés des Pays de la Loire, ainsi que sur l'importance des contraintes professionnelles $[8,9,17,18]$. La prévalence des TMS diagnostiqués est très élevée puisque près de $13 \%$ des salariés présentaient le jour de la visite médicale du travail au moins un TMS-MS. Contrairement à ce que montrent les statistiques d'indemnisation des maladies professionnelles, le TMS-MS le plus fréquemment observé dans cet échantillon n'est pas le syndrome du canal carpien, mais le syndrome de la coiffe des rotateurs.

\section{Le recueil des données cliniques}

Le recueil des données cliniques a reposé à la fois sur un autoquestionnaire pour les symptômes et sur un examen clinique standardisé réalisé par le médecin du travail. Celuici permet d'accroître la valeur diagnostique de l'étude par 
rapport aux grandes enquêtes par autoquestionnaire ou basées sur les statistiques médicolégales [19,12]. L'autoquestionnaire s'inspire directement du questionnaire dit " nordique " dont la validité pour la surveillance des TMSMS a été étudiée [3,4]. Il est comparable dans sa structure aux questionnaires utilisés dans les grandes études internationales sur les TMS, ce qui facilite les comparaisons entre pays [12] L'examen clinique standardisé permet l'analyse de la sévérité des symptômes TMS-MS, puis la recherche standardisée des principaux TMS à l'aide de manœuvres cliniques réalisables en médecine du travail. Aucun examen complémentaire n'est nécessaire, y compris pour établir le diagnostic de syndrome du canal carpien et de syndrome du tunnel cubital. Le guide clinique Saltsa [20], que nous avons traduit en français, est sans doute le guide clinique le plus abouti actuellement [12]. II n'a pas encore été évalué sur le plan métrologique, mais il est proche dans sa conception et sa rédaction des critères de Southampton [22] qui possèdent de bonnes qualités métrologiques [14].

La surveillance des TMS dans les Pays de la Loire a montré le caractère opérationnel du consensus Saltsa pour standardiser le recueil des données cliniques et d'exposition à l'échelle populationnelle en santé au travail. Les modalités de recueil des données cliniques, notamment les principes de l'arbre diagnostique et du guide clinique sous forme de tableaux et de photographies, sont applicables aux spécificités de l'examen clinique en santé au travail. Les critères diagnostiques sont simples et pratiques. Peu d'effet " médecin " a été observé lors du recueil des données entre 2002 et 2004 [17]. La démarche clinique a été accueillie très favorablement par les médecins du travail du réseau. Elle nécessite une formation préalable afin d'homogénéiser les pratiques cliniques et d'assurer sa qualité et son objectivité.

Ainsi, le bilan de la phase pilote (2002-2004) de surveillance des TMS montre que la démarche clinique retenue est applicable à la surveillance épidémiologique des TMS par les services de santé au travail. Un suivi des salariés inclus lors de cette phase pilote de surveillance est mis en œuvre depuis 2007, mobilisant près de 200 médecins du travail formés à ce protocole clinique (cohorte des salariés ligériens baptisée Cosali).

Nous ne discuterons pas ici des critères diagnostiques des différents TMS-MS retenus pour le consensus Saltsa par le groupe d'experts internationaux après analyse de la littérature internationale et permettant d'envisager une homogénéisation du diagnostic des TMS-MS au niveau européen [20].

\section{La méthodologie de recueil des données d'exposition}

Concernant la méthodologie de recueil des données d'exposition retenue par le programme pilote de surveillance des TMS, l'autoévaluation par le salarié des contraintes des situations de travail et de l'exposition aux facteurs de risque de TMS-MS est plus fiable que les seuls intitulés de métiers souvent utilisés pour la surveillance épidémiologique des TMS [19]. L'autoévaluation par questionnaire n'introduit pas de biais de mesure majeur des principaux facteurs de risque biomécaniques par rapport à un interrogatoire sur une journée typique de travail [21]. L'évaluation des contraintes par le médecin du travail aurait pu être utile, notamment pour évaluer la validité de l'autoévaluation, en particulier sur les modalités de l'organisation du travail [1]. Cependant, cette méthodologie aurait considérablement alourdi le protocole de surveillance en entreprise et n'a pas été retenue. Le recueil des données d'exposition au risque par observation des postes de travail n'est pas applicable à la surveillance épidémiologique des TMS, compte tenu de la charge de travail que cela entraînerait pour les médecins du travail $[16,6]$. L'autoquestionnaire utilisé est compréhensible par la très grande majorité des salariés et ses qualités métrologiques sont satisfaisantes [5]. II a cependant été jugé par de nombreux médecins relativement long à remplir, ce qui peut poser des problèmes pratiques d'organisation du recueil des données dans la salle d'attente du cabinet médical ou du véhicule du service médical. Néanmoins, le recueil des données d'exposition par autoquestionnaire reste nettement plus simple à mettre en œuvre que l'observation systématique des situations de travail à l'aide de listes de contrôle (6).

\section{Conclusion}

L'utilisation du consensus Saltsa par le programme pilote de surveillance épidémiologique des Pays de la Loire a permis de disposer d'une évaluation de l'état de santé musculo-squelettique des salariés utilisant une méthodologie qui pourrait être commune à d'autres pays de l'Union européenne. C'est une avancée importante dans la connaissance épidémiologique des TMS par rapport aux études basées sur les statistiques de maladies professionnelles qui sont peu comparables d'un pays à l'autre.

Les salariés vieillissants, comme les salariés plus jeunes, sont exposés à des degrés d'intensité divers à un cumul de contraintes biomécaniques, psychosociales et organisationnelles, ce qui souligne la nécessité d'une approche globale de la prévention dans les entreprises pour réduire l'exposition au risque et assurer le maintien et/ou le retour au travail des salariés souffrant de TMS. Cela nécessite une forte mobilisation de l'ensemble des acteurs de la prévention des risques professionnels (entreprises, partenaires sociaux, pouvoirs publics) et la mise en place d'une politique structurée et coordonnée de prévention des TMS dans les entreprises et les services de santé au travail.

\section{Remerciements}

Nous remercions les médecins du travail et les services de santé au travail des Pays de la Loire qui ont participé à cette étude : Docteurs 
Abonnat, Banon, Bardet, Benetti, Becquemie, Bertin, Bertrand, Bidron, Biton, Bizouarne, Boisse, Bonamy, Bonneau, Bouguer, BouguerDiquelou, Bourut-Lacouture, Breton, Caillon, Cesbron, Chisacoff, Chotard, Compain, Coquin-Geogeac, Cordes, Couet, Coutand, Daniellou, Darcy, Davenas, De Lescure, Delansalut, Dupas, Evano, Fontaine, Frampas-Chotard, Guiller, Guillimin, Harinte, Harrigan, Hervio, Hirigoyen, Jahan, Joliveau, Jube, Kalfon, Laine-Colin, Laventure, Le Dizet, Lechevalier, Leclerc, Ledenvic, Leroux, LeroyMaguer, Levrard, Levy, Logeay, Lucas, Mallet, Martin, Mazoyer, Meritet, Michel, Migne-Cousseau, Moisan, Page, Patillot, Pinaud, Pineau, Pizzala, Plessis, Plouhinec, Raffray, Roussel, Russu, Saboureault, Schlindwein, Soulard, Thomson, Treillard, Tripodi.

\section{Références}

[1] Burdof A, Van der Beek A. Exposure assessment strategies for work-related risk factors for musculoskeletal disorders. Scand J Work Environ Health 1999;25(Suppl. 4):25-30.

[2] Chiron E, Roquelaure $\mathrm{Y}, \mathrm{Ha}$ C, et al. Les TMS et le maintien en emploi des salariés de 50 ans et plus : un défi pour la santé au travail et la santé publique. Sante Publique 2008;(Suppl. 3):S19-28.

[3] Descatha A, Roquelaure Y, Aublet-Cuvelier A, et al. Le questionnaire de type " nordique " : intérêt dans la surveillance des pathologies d'hypersollicitation du membre supérieur d'origine professionnelle. Documents pour le médecin du travail, $\mathrm{n}^{\circ} 112,4^{\mathrm{e}}$ trimestre 2007.

[4] Descatha A, Roquelaure Y, Chastang JF, et al. Validity of Nordicstyle questionnaires in the surveillance of upper-limb workrelated musculokeletal disorders. Scand J Work Environ Health 2007;33(1):58-65.

[5] Descatha A, Roquelaure $Y$, Evanoff B, et al. Selected questions on biomechanical exposures for surveillance of upper-limb work-related musculoskeletal disorders. Int Arch Occup Environ Health 2007;81:1-8.

[6] Descatha A, Roquelaure Y, Caroly S, et al. Self-administered questionnaire and direct observation by checklist: Comparing two methods for physical exposure surveillance in a highly repetitive tasks plant. Appl Ergon 2009;40:194-8.

[7] Ha C, Roquelaure Y. Réseau expérimental de surveillance épidémiologique des troubles musculo-squelettiques dans les Pays de la Loire. Protocole de la surveillance dans les entreprises (2002-2004). Mai 2007, 84 pages. http:// www.invs.sante.fr/publications/2007/protocole_tms_loire/ protocole tms loire.pdf.

[8] Ha C, Roquelaure Y, Leclerc A, et al. The French Musculoskeletal Disorders Surveillance Program: Pays de la Loire network. Occup Environ Med 2009;66:471-9.
[9] Ha C (coordinateur). Numéro thématique. TMS d'origine professionnelle : une préoccupation majeure Bull Epidemiol Hebd 2010;5(6):33-56.

[10] Halpern $M$, Hiebert R, Nordin $M$, et al. The test-retest reliability of a new occupational risk factors questionnaire for outcome studies of low back pain. Applied Ergon 2001;32:39-46.

[11] Kuorinka I, Jonsson B, Kilbom A, et al. Standardised Nordic questionnaire for the analysis of musculoskeletal symptoms. Applied Ergonomics 1987;18(3):233-7.

[12] Leclerc A. Les TMS du membre supérieur : quelques données épidémiologiques. In: Lasfargues $G$, Roquelaure $Y$, Fouquet $B$, Leclerc A, editors. Pathologie d'hypersollicitation périarticulaires des membres supérieurs. Paris: Masson; 2003. p. 3-26.

[13] Meyer J-P, Sluiter J, Rest K, et al. Troubles musculosquelettiques du membre supérieur liés au travail. Consensus clinique pour le repérage des formes précoces de TMS. Arch Mal Prof 2002;63: 32-45.

[14] Palmer KT, Walker-Bone KE, Linaker C, et al. The Southampton examination schedule for the diagnosis of musculoskeletal disorders of the upper limb. Ann Rheum Dis 2000;59:5-11.

[15] Roquelaure $Y$, Touranchet A, Ha C. Réseau expérimental de surveillance épidémiologique des troubles musculo-squelettiques (TMS) d'origine professionnelle. Rapport final pour l'institut de veille sanitaire, Saint Maurice, 2001, $83 \mathrm{p}$.

[16] Roquelaure Y, Mariel J, Fanello S, et al. Active epidemiological surveillance of work-related musculoskeletal disorders (WMSDs) in a shoe factory. Occup Environ Med 2002;59: 452-8.

[17] Roquelaure $\mathrm{Y}, \mathrm{Ha} \mathrm{C}$, Leclerc A, et al. Epidemiological surveillance of upper extremity musculoskeletal disorders in the working population: the French Pays de la Loire study. Arthritis Rheum 2006;55:765-78.

[18] Roquelaure Y, Ha C, Rouillon C, et al. Risk factors for upperextremity musculoskeletal disorders in the working population. Arthritis Rheum 2009;61:1425-34.

[19] Silverstein B, Viikari-Juntura E, Kalat J. Use of the prevention index to identify industries at high risk of work-related musculoskeletal disorders of the neck, back, and upper extremities in Washington state, 1990-1998. Am J Ind Med 2002;41:14969.

[20] Sluiter JK, Rest KM, Frings-Dresen MHW. Criteria document for evaluating the work-relatedness of upper extremity musculoskeletal disorders. Scand J Work Environ Health 2001;27(Suppl. 1): 1-102.

[21] Viktorin C, Vingaed E, Mortimer $M$, et al. Interview versus questionnaire for assessing physical loads in the populationbased Music-Norrtalje Study. Am J Ind Med 1999;35:441-55.

[22] Walker-Bone KE, Palmer KT, Reading I, et al. Criteria for assessing pain and non articular soft-tissue rheumatic disorders of the neck and upper limb. Semin Arthritis Rheum 2003;33:16884 . 